\title{
Version Date
}

National Cancer Institute

\section{Source}

National Cancer Institute. Version Date. NCI Thesaurus. Code C93702.

The date (and time) on which an entity is versioned. 